\title{
Review of techniques for ultrasonic determination of kidney stone size
}

This article was published in the following Dove Press journal: Research and Reports in Urology

\author{
Mohankumar Vijayakumar \\ Arvind Ganpule \\ Abhishek Singh \\ Ravindra Sabnis \\ Mahesh Desai \\ Department of Urology, Muljibhai \\ Patel Urological Hospital, Nadiad, \\ Gujarat,India
}

Correspondence: Arvind Ganpule Department of Urology, Muljibhai Patel Urological Hospital, Dr. Virendra Desai Road, Nadiad, Gujarat 38700I, India Tel +9l $9824 \mid 88685$

Email doctorarvindI@gmail.com
Introduction: Imaging is a vital $\operatorname{cog}$ in the wheel of diagnosis and management of patients suspected with renal and ureteric calculi, and it is imperative to choose the appropriate investigation that is accurate as well as safe for the patient. At present, computed tomography (CT) is the gold standard for a patient suspected to have stone disease. However, CT scan is associated with the hazards of radiation and high cost. Ultrasonography (US) is cheap and also devoid of any radiation hazard to the patient. But, at the same time, its usage is limited by decreased sensitivity and specificity, inaccuracy in measuring stone size, and observer dependency. In this article, we review the techniques to improve the accuracy of US in measuring stone size.

Accuracy of US: According to a review, the sensitivity and specificity for renal calculi are $45 \%$ and $88 \%$, respectively, and for ureteric calculi, they are $45 \%$ and $94 \%$, respectively. The sensitivity of US decreased when the size of the stone is $<3 \mathrm{~mm}$ and also in a nondilated system, and the sensitivity increased as the size of the stone increased.

Tools to improve accuracy: There are factors that can be adjusted to increase the accuracy of stone measurement. The main factors are changes in gain and depth and alternate modes such as flash angle imaging, harmonic mode, and S mode. Also measures such as use of shadow for size measurement can help in improving the accuracy of stone size measurement. A new automated computerized stone-sizing program improves the accuracy of stone size calculation and reduces user variability.

Conclusion: US is an ideal first-line imaging modality for nephrolithiasis due to its advantages such as low cost, absence of radiation, and easy availability. However, the only limitation is its reduced sensitivity and specificity when compared with $\mathrm{CT}$. The addition of newer modes can improve the accuracy in stone size measurement.

Keywords: ultrasonography, accuracy, stone size, S mode, acoustic shadow

\section{Introduction}

Imaging is a vital cog in the wheel of diagnosis and management of patients suspected with renal and ureteric calculi, and it is imperative to choose the appropriate investigation that is accurate and safe for the patient. The choice of appropriate investigation depends on various factors such as the nature of presentation, body habitus of the patient, cost of the investigation, and the effect of the investigation on the patient. Many modalities are available for investigating stone in patients such as ultrasonography (US), X-ray, computed tomography (CT) scan, and magnetic resonance imaging (MRI). CT, US, and X-ray are used widely. US has lesser sensitivity when compared with CT for diagnosing renal stones. ${ }^{1-4}$ 
US has also been considered inferior to CT in the assessment of the size of the stones. ${ }^{1-4}$ US tends to overestimate the size of stones, particularly the smaller ones. ${ }^{5}$ This has huge implications in management decisions with respect to smaller calculi. Overestimation of stone size leads to surgery for stones which would have been managed conservatively had the accurate size of the stone known previously. It is imperative that the surgeon knows the accurate stone size preoperatively for decision making and also patient counseling.

At present, $\mathrm{CT}$ is the gold standard for a patient suspected to have stone disease. ${ }^{6}$ However, CT scan is associated with the hazards of radiation and high cost. US is cheap and also devoid of any radiation hazard to the patient. But, at the same time, its usage is limited by decreased sensitivity and specificity, inaccuracy in measuring stone size, and observer dependency. In this article, we review the techniques to improve the accuracy of US in measuring stone size.

\section{Principles of US}

The transducer delivers acoustic energy in the form of short pulses to the patient. These waves propagate through the tissues and are reflected back to the source. These waves pass through tissues of variable densities and acoustic impedances on their way back. The images generated by the receiver depend on the amplitude as well as the travel time of the reflected waves. The common image modality used is the gray-scale image, and it is known as B mode (brightness mode) in which the stones appear bright with a dark shadow distally. The B mode US detects stone by making use of the differences between the surrounding tissue and the stone. The harmonic mode in B mode US can increase the stone detection by improving the resolution and decreasing the clutter. The stones reflect the waves strongly, which causes the stones to appear bright and leads to increase in echogenicity. The post-acoustic shadow beyond the stone is usually due to the inability of the waves to penetrate the stone.

The probe used for renal or ureteric calculi is usually 3.5-5 $\mathrm{MHz}$ probe. The patient is usually in supine position for imaging the right kidney. The probe is placed in the midaxillary line in the right lower intercostal space. The probe is aimed posteriorly and the liver is used as an acoustic window on the right side. The kidney is imaged in both the longitudinal and transverse axes. The patient is in supine or right lateral position in the left side.

\section{Accuracy of US}

The sensitivities and specificities differ across studies due to the difference in the body habitus, the reference standards used, and inter-observer variations. The sensitivity and specificity for renal calculi are $45 \%$ and $88 \%$, respectively, and for ureteric calculi, they are $45 \%$ and $94 \%$, respectively. ${ }^{3}$ The sensitivity of US decreases when the size of the stone is $<3$ $\mathrm{mm}$ and also in a nondilated system. The study by Fowler et $\mathrm{al}^{1}$ demonstrated a sensitivity and specificity of $24 \%$ and $90 \%$ for stones of any location. Unal et $\mathrm{al}^{7}$ demonstrated a sensitivity of $69 \%$ and a specificity of $87 \%$ for stones of any location. They also found that the sensitivity increased as the size of the stone increased. The sensitivity was as low as $13 \%$ for stones $<3 \mathrm{~mm}$ and as high as $71 \%$ for stones $>7 \mathrm{~mm}$ in size.

US usually overestimates the stone size. Ray et $\mathrm{al}^{3}$ found that US overestimates the stone size by $1.8 \mathrm{~mm}$ on average. The overestimates of the stone size were by an average of 1.5 $\mathrm{mm}$ in Fowler et al ${ }^{1}$ and $1.5-2 \mathrm{~mm}$ in Dunmire et al. ${ }^{8}$ Studies have also reported an underestimation of renal calculi by US. ${ }^{2}$

\section{Tools to improve accuracy}

Accurate estimation of stone size has major implications in clinical decision making. There are factors that can be adjusted to increase the accuracy of stone measurement. The main factors that help in improving the accuracy are changes in gain and depth and alternate modes such as flash angle imaging and $\mathrm{S}$ (stone-specific) mode. A new automated computerized stone-sizing program improves the accuracy of stone size calculation and reduces user variability.

\section{Gain and depth}

The US overestimated the size of the stone with an increase in depth and also an increase in gain at a given depth. So the accuracy of estimation of stone size improves with accurate adjustment of depth and gain. ${ }^{22}$

\section{Automated computerized algorithm}

Dunmire et $\mathrm{a}^{22}$ demonstrated that the use of computerized algorithms for the measurement of stone size increased its accuracy.

\section{Harmonic imaging}

Harmonic imaging is very similar to that of ray-line imaging but the principle is different in that the image is built from signals of higher frequency in order to improve the lateral resolution. The beam is made more uniform across depths by keeping the focus much deeper to the stone. Studies have shown that harmonic imaging improves the accuracy of stone measurement.

\section{$S$ mode}

Cunitz et $\mathrm{al}^{26}$ proposed a new mode in US termed as S mode or stone-specific mode which helps in identifying the difference 
between the stone and the surrounding structures in an accurate way. The settings for S mode US are increased frequency and a high scanning line density (100 lines/frame). This results in reduction of the twinkling artifact caused by the microbubbles and improves the resolution of the image. The $\mathrm{S}$ mode US increases the sensitivity compared with the traditional $\mathrm{B}$ mode..$^{22,26}$ The sensitivity was $78 \%$ for $\mathrm{S}$ mode vs. $61 \%$ for traditional US. Measurement of stone size and shadow size under $\mathrm{S}$ mode showed results similar to CT. Most of the current US machines can incorporate the $\mathrm{S}$ mode without any modification.

\section{Acoustic shadow width}

The use of acoustic shadow width has recently been studied and has been proven to improve the accuracy of stone measurement. Dunmire et al, ${ }^{23}$ in their recent study, the authors found that measuring of the width of the stone shadow was a more accurate measure than measuring the actual stone on US, and this method improves the accuracy to near CT levels. They measured the area behind the stone where the waves were absent instead of the front. Using this method, stone size was estimated with significantly greater accuracy, regardless of whether ray line, spatial compound, or harmonic imaging was used. Indeed, accuracy of sizing to within $1 \mathrm{~mm}$ was obtained in $78 \%$ of cases.

\section{Twinkling artifact}

The twinkling artifact appears as a rapidly alternating color Doppler signal that imitates turbulent flow, often identified behind a strongly reflecting stationary irregular interface, such as a renal calculus. It is the appearance of colors in a mixed pattern in Doppler. However, a Doppler spectrum of the area of twinkling shows only a heterogeneous broadband aliasing signal consistent with noise produced by the reflected signal. The phenomenon was first described by Rahmouni et $\mathrm{al}^{9}$ as an artifact generated by a strongly reflecting medium composed of small individual reflectors. The twinkling artifact occurs with urinary tract calculi and parenchymal calcifications, but it can also be observed with noncalcified biliary calculi and any material with an irregular, rough, or reflective surface. The twinkling artifact, when imaged with a high-pulse repetition frequency, can show nonobstructing calculi as well as many obstructive calculi. Evaluation of the twinkling artifact is a complementary technique to standard gray-scale shadowing of calculi and improves detection of urolithiasis on sonography.

\section{US vs. CT}

Non-contrast computerized tomography (NCCT) is currently the gold standard for diagnosing nephrolithiasis with a sensitivity and specificity of $94 \%-97 \%$ and $96 \%-100 \%$, respectively, when compared with US which has a sensitivity of $40 \%$ and a specificity of $84 \%$ and plain abdominal radiography or intravenous urography which has a sensitivity and specificity of $45 \%-58 \%$ and $52 \%-87 \%$, respectively. ${ }^{1,10-13}$ NCCT has got some advantages over US that it is not influenced by the body habitus and the accuracy is not interfered by bowel gas. Almost all the stones except some matrix stones and indinavir stones can be detected by NCCT accurately with reliable size measurements with the error in stone size being around $3.6 \%{ }^{14}$ The main disadvantages of NCCT are that it is costly and also has a risk of radiation exposure, the cumulative effects of which is hazardous and has also been found to lead to malignancy later. The International Commission on Radiation Protocol (ICRP) recommends that exposure should not exceed $20 \mathrm{mSv}$ per year over a 5-year period or it should not exceed $50 \mathrm{mSv}$ in any single year. ${ }^{15}$ The average radiation dose the patient is exposed in a single NCCT of the abdomen and pelvis is $15 \mathrm{mSv}$. The patient is exposed to $3 \mathrm{mSv}$ with a low-dose protocol and to $1-3 \mathrm{mSv}$ with an ultralow-dose protocol. It has been estimated that $\sim 1$ in 1400 patients with age 60 undergoing NCCT would develop a radiation-induced solid cancer or leukemia. ${ }^{16}$ There is also evidence in literature about the average number of CTs a patient with nephrolithiasis undergoes. ${ }^{17}$ Studies indicate that repeated CT scans expose patients to more than the allowed limit of radiation exposure. ${ }^{12,18} \mathrm{US}$ is a urinary tract imaging modality that has no risk of radiation exposure or risk of subsequent attributable malignancy. It has also got the advantages of being quick, inexpensive, and repeatable. The low sensitivity of US compared with NCCT suggests that it has poor utility for the exclusion of urolithiasis. Delineation of stones on US often necessitates acoustic shadowing, and a lack of shadowing may occur from intervening tissue with different acoustic impedance values, filling of the shadow from reverberation, or incorrect selection of transducer settings. Identification of stones $<4 \mathrm{~mm}$ was most limited on US. Visualization of the right renal system is easier because of improved acoustic windows from the liver and less need for intercostal scanning because of the inferior position of the kidney. Correlation between US and NCCT findings decreased with smaller stone size and ureteral location and increased with right-sided laterality.

\section{Advantage of US over CT}

The main advantage of US over CT is its reduced cost, absence of radiation, and portability. The effects of these radiations are cumulative, and the cumulative exposure 
has the risk of future malignancies. The effect accumulates throughout life, and care should be taken to avoid radiation in younger people and pregnant women. In a multicentric comparative effectiveness trial, Smith-Bindman et $\mathrm{al}^{24}$ has concluded that US was associated with lower radiation exposure without significant impact on diagnosis and serious adverse events and rehospitalization rates when compared with CT.

US has advantages in pediatric population because of the reduced skin to probe distance in children. US is recommended as the firstline imaging modality for patients who are pregnant and pediatric patients $(<14$ years old $) .{ }^{25}$ SmithBindman etal ${ }^{24}$ have published the STONE trial, in which they randomized patients with suspected obstructive nephrolithiasis to evaluation with CT, US performed at the bedside, or US performed in the radiology department. Overall, 2759 patients were randomized to one of the three arms and then assessed over 180 days to evaluate the accuracy of stone diagnosis, based on patient reports of stone passage or surgical intervention. Overall, no significant differences were reported in sensitivity $(\sim 85 \%)$, specificity $(\sim 50 \%)$, or complications between the three arms at the time of discharge from the emergency department.

\section{Ureteric vs. renal calculi}

NCCT is superior over US for the evaluation of ureteral calculi. Yilmaz et $\mathrm{al}^{12}$ have proven that NCCT is superior to US for the detection of ureteral calculi with a sensitivity of $94 \%$ for NCCT in comparison with $19 \%$ for US. The same cannot be said for renal calculi, as US has got a sensitivity comparable to CT in renal calculi (Table 1).

\section{Pediatric urolithiasis}

NCCT is currently the method of choice for diagnosing urinary tract calculi because of its increased sensitivity and specificity, and detail. The sensitivity of US in pediatric population for renal calculi was higher compared with ureteric calculi. There is evidence in literature comparing the

Table I Detection of urinary tract calculi by US

\begin{tabular}{llll}
\hline Study & N & Sensitivity & Specificity \\
\hline Ureteric calculi & & & \\
Yilmaz et al $^{12}$ & 97 & 19 & 97 \\
Sheafor et al $^{13}$ & 45 & 61 & 100 \\
Hamm et al $^{19}$ & 125 & $1 \mathrm{I}$ & 97 \\
Unal et al $^{7}$ & 137 & 69 & 87 \\
Renal calculi $_{\text {Fowler et al }}^{1}$ & 188 & 24 & \\
Unal et al $^{7}$ & 137 & 69 & 90 \\
Ulusan et al $^{20}$ & 101 & 44 & 87 \\
\hline Abbreviation & & & 82 \\
\hline
\end{tabular}

Abbreviation: US, ultrasonography. accuracy of US and CT in pediatric nephrolithiasis. Eshed and Witzling ${ }^{21}$ opined that CT failed to provide any significant information if performed after a normal ultrasound. So it is better to use ultrasound as a first line of investigation in pediatric population and reserve CT in cases where US is inconclusive.

\section{Conclusion}

US is an ideal first-line imaging modality for nephrolithiasis due to its advantages such as low cost, absence of radiation, and easy availability. But the reason for its limited use is due to its decreased sensitivity and reduced accuracy in measuring the stone size, the areas in which CT scores over US. The addition of newer modes in US and the change in settings have increased the accuracy of US but not to the extent that it can replace CT. European Association of Urology also recommends US as the first-line investigation for patients who present with suspected nephrolithiasis. ${ }^{27}$ So, for these reasons, US can be considered as first line in selected situations but it is far from being called as an ideal investigation for nephrolithiasis.

\section{Disclosure}

The authors report no conflicts of interest in this work.

\section{References}

1. Fowler KAB, Locken JA, Duchesne JH, Williamson MR.US for detecting renal calculi with nonenhanced $\mathrm{CT}$ as a reference standard. Radiology. 2002;222(1):109-113.

2. Kanno T, Kubota M, Sakamoto H, et al. The efficacy of ultrasonography for the detection of renal stone. Urology. 2014;84(2):285-288.

3. Ray AA, Ghiculete D, Pace KT, Honey RJ. Limitations to ultrasound in the detection and measurement of urinary tract calculi. Urology. 2010;76(2):295-300

4. Wong LM, Jenkins M. Accuracy of ultrasonography for the evaluation of urolithiasis in patients undergoing extracorporeal shockwave lithotripsy: comparison with non-contrast computed tomography. In: American Urological Association Annual Meeting Abstract, San Diego, CA; 2016

5. Miller O, Kane C. Time to stone passage for observed ureteral calculi: a guide for patient education. J Urol. 1999;162:688-691.

6. Fwu C, Eggers P, Kimmel P, Kusek JW, Kirkali Z. Emergency room visits, use of imaging and drugs for urolithiasis have increased in the United States. Kidney Int. 2013;83:479-486.

7. Unal D, Yeni E, Karaoglanoglu M, Verit A, Karatas OF. Can conventional examinations contribute to the diagnostic power of unenhanced helical computed tomography in urolithiasis? Urol Int. 2003;70(1):31-35.

8. Dunmire B, Lee FC, Hsi RS, Cunitz BW, Paun M, Bailey MR, et al. Tools to improve the accuracy of kidney stone sizing with ultrasound. J Endourol Endourol Soc. 2015;29(2):147-152.

9. Rahmouni A. Bargoin R, Herment A, Bargoin N, Vasile N. Color Doppler twinkling artifact in hyperechoic regions.Radiology. 1996;199:269-271.

10. Smith RC, Verga M, McCarthy S, Rosenfield AT. Diagnosis of acute flank pain: value of unenhanced helical CT. AJR Am J Roentgenol. 1996;166:97-101.

11. Miller OF, Rineer SK, Reichard SR, et al. Prospective comparison of unenhanced spiral computed tomography and intravenous urogram in the evaluation of acute flank pain. Urology. 1998;52:982-987. 
12. Yilmaz S, Sindel T, Arslan G, et al. Renal colic: comparison of spiral CT, US, and IVU in the detection of ureteral calculi. Eur Radiol. 1998;8: 212-217.

13. Sheafor DH, Hertzberg BS, Freed KS, et al. Non-enhanced helical CT and US in the emergency evaluation of patients with renal colic: prospective comparison. Radiology. 2000;217:792-797.

14. Olcott EW, Sommer FG, Napel S. Accuracy of detection and measurement of renal calculi: In-vitro comparison of three dimensional spiral CT, radiography, and nephrotomography. Radiology. 1997;204:19-25.

15.1990 recommendations of the international commission on radiological protection. AnnICRP.1991;21(1-3):1-201.

16. Smith-Bindman R, Lipson J, Marcus R, et al. Radiation dose associated with common computed tomography examinations and the associated lifetime attributable risk of cancer. Ann Intern Med. 2009;169:2078-2086.

17. Romero V,Akpinar H, Assimos DG. Kidney stones: a global picture of prevalence, incidence, and associated risk factors. Rev Urol. 2010;12:e86-e96.

18. Ferrandino MN, Bagrodia A, Pierre SA, et al. Radiation exposure in the acute and short-term management of urolithiasis at 2 academic centers. J Urol. 2009;181:668-673.

19. Hamm M, Wawroschek F, Weckermann D, et al. Unenhanced helical computed tomography in the evaluation of acute flank pain. Eur Urol. 2001;39:460-465.
20. Ulusan S, Koc Z, Tokmak N. Accuracy of sonography for detecting renal stone: comparison with CT. J Clin Ultrasound. 2007;35: 256-261.

21. Eshed I, Witzling M. The role of unenhanced helical CT in the evaluation of suspected renal colic and atypical abdominal pain in children. Pediatr Radiol.2002;32:205.

22. Dunmire B, Lee FC, Hsi RS, et al. Tools to improve the accuracy of kidney stone sizing with ultrasound. J Endourol. 2015;29(2): 147-152.

23. Dunmire B, Harper JD, Cunitz BW, et al. Use of the acoustic shadow width to determine kidney stone size with ultrasound. J Urol. 2016;195(1):171-177.

24. Smith-Bindman R, Aubin C, Bailitz J, et al. Ultrasonography versus computed tomography for suspected nephrolithiasis. $N$ Engl J Med. 2014;371:1100-1110.

25. Brisbane W, Bailey MR, Sorensen MD. An overview of kidney stone imaging techniques. Nat Rev Urol. 2016;13(11):654-662.

26. Cunitz B, Dunmire B, Paun M, et al. Improved detection of kidney stones using an optimized Doppler imaging sequence. IEEEIntUltrasonSymp.2014;2014:452-455.

27. Türk C, Petř́ik A, Sarica K, et al. EAU guidelines on interventional treatment for urolithiasis. Eur Urol. 2016;69:475-482.
Research and Reports in Urology

\section{Publish your work in this journal}

Research and Reports in Urology is an international, peer-reviewed, open access journal publishing original research, reports, editorials, reviews and commentaries on all aspects of adult and pediatric urology in the clinic and laboratory including the following topics: Pathology, pathophysiology of urological disease; Investigation and treatment of

\section{Dovepress}

urological disease; Pharmacology of drugs used for the treatment of urological disease. The manuscript management system is completely online and includes a very quick and fair peer-review system, which is all easy to use. Visit http://www.dovepress.com/testimonials.php to read real quotes from published authors. 